33. Pandhi RK, Kumar AS, Satish DA, Bhutani LK (1984) Fixed drug eruptions on male genitalia: clinical and etiologic study. Sex Transm Dis 11:164-166

34. Schlosser BJ (2010) Contact dermatitis of the vulva. Dermatol Clin 28:697-706

35. Sell MB (1985) Sensitization to thioridazine through sexual intercourse. Am J Psychiatry 142:271-272

36. Shah A, Panjabi C (2004) Human seminal plasma allergy: a review of a rare phenomenon. Clin Exp Allergy 34:827-838

37. Sharma VK, Dhar S, Gill AN (1996) Drug related involvement of specific sites in fixed eruptions: a statistical evaluation. J Dermatol 23:530-534

38. Steensma DP (2003) The kiss of death: a severe allergic reaction to a shellfish induced by a goodnight kiss. Mayo Clin Proc 78:221-222

39. Uter W, Geier J, Bauer A, Schnuch A (2013) Risk factors associated with methylisothiazolinone contact sensitization. Contact Dermatitis 69:231-238

40. Vermaat $H$, Smienk F, Rustemeyer T, Bruynzeel DP, Kirtschig G (2008) Anogenital allergic contact dermatitis, the role of spices and flavour allergy. Contact Dermatitis 59:233-237

41. Vermaat $H$, van Meurs $T$, Rustemeyer T, Bruynzee DP, Kirtschig G (2008) Vulval allergic contact dermatitis due to peppermint oil in herbal tea. Contact Dermatitis 58:364-365

42. Wain EM, Neill S (2008) Fixed drug eruption of the vulva secondary to fluconazole. Clin Exp Dermatol 33:784-785

43. Warshaw EM, Furda LM, Maibach HI, Rietschel RL, Fowler JF Jr, Belsito DV, Zug KA, DeLeo VA, Marks JG Jr, Mathias CG, Pratt MD, Sasseville D, Storrs FJ, Taylor JS (2008) Anogenital dermatitis in patients referred for patch testing: retrospective analysis of cross-sectional data from the North American Contact Dermatitis Group, 1994-2004. Arch Dermatol 144:749-755

44. Weidinger S, Mayerhofer A, Raemsch R, Ring J, Kohn FM (2006) Prostate-specific antigen as allergen in human seminal plasma allergy. J Allergy Clin Immunol 117:213-215

45. Weidinger S, Ring J, Kohn FM (2005) IgE-mediated allergy against human seminal plasma. Chem Immunol Allergy 88:128-138

46. Winter UM, Harth W, Treudler R, Simon JC (2008) Typ-I-Allergie auf Seminalplasma. Hautarzt 59:649-652

Hautarzt $2015 \cdot 66: 52$

DOI 10.1007/s00105-014-3564-y

Online publiziert: 23. Dezember 2014

c) Springer-Verlag Berlin Heidelberg 2014

F. Kleffner · J. Schürholz · S. Burckhardt · C. Mauch · M. Schlaak

Klinik und Poliklinik für Dermatologie und Venerologie, Hauttumorzentrum

im CIO Köln Bonn, Uniklinik Köln, Köln, Deutschland

\title{
Erratum zu: Merkel-Zell-Karzinom
}

In - Tab. 2 war leider die letzte Tabellenspalte falsch benannt. Wir bitten Sie, die korrekte Version von - Tab. 2 zu beachten. Wir bitten, den Fehler zu entschuldigen.

Die Redaktion

\section{Korrespondenzadresse}

PD Dr. M. Schlaak

Klinik und Poliklinik für Dermatologie und Venerologie, Hauttumorzentrum im ClO Köln Bonn Uniklinik Köln, Kerpener Str. 62 50937 Köln max.schlaak@uk-koeln.de
Tab. 2 Immunhistochemische Marker des Merkel-Zell-Karzinoms (MCC) und seiner Differenzialdiagnosen. (Nach Becker et al. [32])

$\begin{array}{lll}\text { MCC } & \begin{array}{l}\text { Kutanes } \\ \text { Lymphom }\end{array} & \begin{array}{l}\text { Malignes } \\ \text { Melanom }\end{array}\end{array}$

Kutane Metastasen eines kleinzelligen Karzinoms der Lunge

\begin{tabular}{lllll}
\hline Zytokeratin-20 (CK20) & + & - & - & - \\
\hline $\begin{array}{l}\text { Neuronenspezifische } \\
\text { Enolase (NSE) }\end{array}$ & + & - & - & $+/-$ \\
\hline Chromogranin A (CgA) & $+/-$ & - & - & + - \\
\hline HIP-1 & + & +- & - & - \\
\hline Vimentin & - & + & + & - \\
\hline Melan A/MART-1 & - & - & + & - \\
\hline $\begin{array}{l}\text { "Leucocyte Common } \\
\text { antigen" (LCA) }\end{array}$ & - & + & - & + \\
\hline $\begin{array}{l}\text { "Thyroidea transcription } \\
\text { factor-1" (TTF-1) }\end{array}$ & - & - & - & \\
\hline
\end{tabular}

